\title{
Behaviour of interfaces in a diffusion-absorption equation with critical exponents
}

\author{
VICTOR A. GALAKTIONOV ${ }^{\dagger}$
}

Department of Mathematical Sciences, University of Bath, Bath BA2 7AY, UK

Keldysh Institute of Applied Mathematics, Miusskaya Sq. 4, 125047 Moscow, Russia

SERGEI I. SHMAREV

Lavrentiev Institute of Hydrodynamics, 630090 Novosibirsk, Russia

Departamento de Matemáticas, Universidad de Oviedo, 33007 Oviedo, Spain

AND

JUAN L. VAZQUEZ ${ }^{\S}$

Departamento de Matemáticas, Universidad Autónoma de Madrid, 28049 Madrid, Spain

[Received 10 September 1999 and in revised form 16 February 2000]

We consider the Cauchy problem for the porous medium equation with strong absorption

$$
u_{t}=\left(u^{m}\right)_{x x}-u^{p} \quad \text { for } \quad x \in \mathbf{R}, \quad t>0,
$$

with continuous compactly supported initial data $u(x, 0)=u_{0}(x) \geqslant 0$ in the critical case $m+p=2$ of the range of parameters $m>1, p<1$. We study the regularity of solutions and interfaces and compare the results with the purely diffusive case $u_{t}=\left(u^{m}\right)_{x x}$. Important differences are found in the interface behaviour and equations, in the occurrence of turning points and inflection points of the interfaces, and in the fact that bounded solutions extinguish in finite time. All these phenomena are examined and described. The critical case studied here allows for a comparatively simpler and richer analysis of the qualitative behaviour of non-linear parabolic equations involving finite propagation $(m>1)$ and strong absorption $(p<1)$.

Keywords: Non-linear diffusion equation, strong absorption, interface equation, regularity, analyticity, turning points, matching expansion

\section{Introduction}

This paper is devoted to the investigation of the question of behaviour and higher regularity of solutions and interfaces of the Cauchy problem for the equation

$$
u_{t}=\left(u^{m}\right)_{x x}-u^{p},
$$

with initial function

$$
u(x, 0)=u_{0}(x) \geqslant 0, \quad x \in \mathbf{R} .
$$

\footnotetext{
†Email: vag@maths.bath.ac.uk

*Email: shmarev@pinon.ccu.uniovi.es

${ }^{\S}$ Email: juanluis.vazquez@uam.es
} 
We consider the range of parameters

$$
m>1, \quad p<1, \quad m+p=2 .
$$

For definiteness, we assume that the initial function $u_{0}$ is continuous, non-negative and bounded. We also assume that it has compact support. Let us briefly examine the consequences of the conditions on the exponents: condition $m>1$ implies slow diffusion, so that a solution with initial compact support will preserve that property in time (finite propagation property), and there is certain separation between the regions $\{u>0\}$ and $\{u=0\}$, called the free boundary, usually formed by a number of curves called interfaces. The regularity of the interfaces is one of the main concerns of this work. Besides, the equation is degenerate parabolic at the level $\{u=0\}$, so that the regularity of the solution near an interface is under question. On the other hand, condition $p<1$ implies strong absorption for small intensities, which means that bounded solutions extinguish in finite time. The combination $m>1$ and $p<1$ also means that the support of the solutions can behave in an expanding or contracting way, in other words the interfaces can move forward or backward in space, thus exhibiting so-called heating and cooling fronts. Such names derive from the typical application of this equation to the description of thermal propagation in absorbing media. This exponent combination can also give rise to pulse splitting, whereby a solution can have a connected support at $t=0$ and lose this property for $t>0$. We refer to the works of Kalashnikov [27] and Rosenau and Kamin [32] for more information about the general problem and to Chen, Mimura and Matano [13] for the basic properties of the interfaces, including continuity and number of connected components of the support. The equation

$$
u_{t}=a\left(u^{m}\right)_{x x}-b u^{p}, \quad a, b>0,
$$

apparently more general than (1.1), can be reduced to it by a simple rescaling. It is interesting to insert these coefficients in the formulae to see how the results for the diffusion-absorption equation bifurcate from the purely diffusive case $a=1, b=0$, i.e. the porous medium equation. These equations are model cases in the general type of diffusion-absorption equations of the form $u_{t}=$ $\Phi(u)_{x x}-F(u)$ with suitable monotone non-decreasing functions $\Phi, F$.

We are interested here in studying the best regularity of the interfaces and of the solutions near an interface, and relating it to the law describing the dynamics of these interfaces. While for $m>1$ the dynamics are given by the famous Darcy law, according to which the velocity of any interface curve equals the gradient of a certain potential function, called the pressure, which is given by formula

$$
v=\frac{m}{m-1} u^{m-1},
$$

this does not hold for $m<1$. A basic analysis of regularity and interface behaviour has been performed in previous papers by the authors for $m+p \geqslant 2$ and $0<m+p<2$ respectively. In the first case, if $m+p>2$, there is an alternative: Darcy's law fails only for cooling waves, being true for heating waves; [18]. On the other hand, the dynamics for $m+p<2$ have nothing to do with Darcy's law, as is proved in [19]. We address here the special properties of the transition case $m+p=2$, where the dynamics of the interfaces are controlled by a modified Darcy law, which for a smooth interface $x=\zeta(t)$ reads

$$
V=z-\frac{a b m}{z},
$$




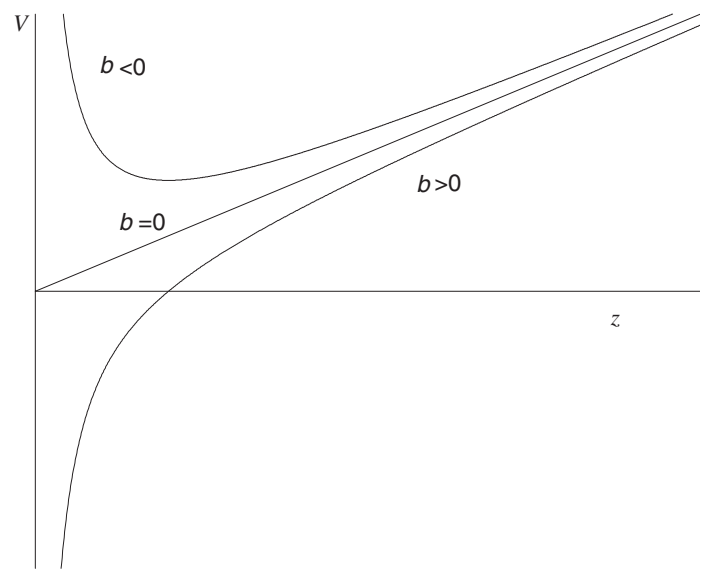

FIG. 1. Darcy's and modified Darcy's laws.

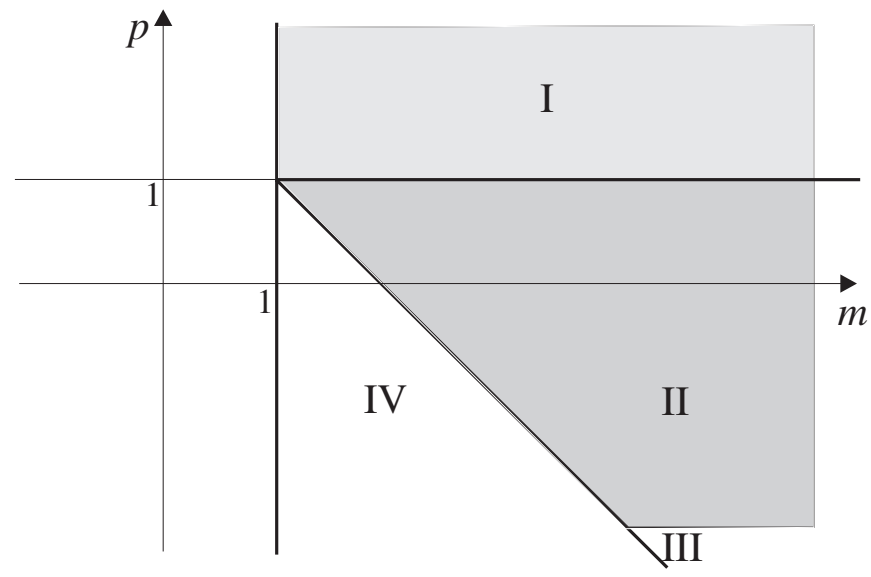

FIG. 2. Different ranges of application of Darcy's law.

where $V$ is the velocity of the interface front (positive if expanding) and $z \geqslant 0$ is the absolute value of the slope of the pressure $v$ calculated at the interface point $(\zeta(t), t)$ as a lateral limit from inside the support of the solution. Thus, for the interface bounding the support on the right-hand side we have $z=-v_{x}(\zeta(t)-, t)$, or in other notation $z=-D_{x}^{-} v(\zeta(t), t)$. In comparison Darcy's law reads $V=z$.

In order to prove the result we must begin with a less regular formula described in (2.7). The case $p+m=2$ is maybe the simplest case where Darcy's law ceases to hold for this family of problems: it admits a more complete and at the time simpler mathematical treatment of the typical phenomena associated to slow diffusion with strong absorption; it allows us to derive strong regularity conclusions; and at the same time marks the transition between quite different types of interface dynamics occurring for $m+p>2$ and $m+p<2$.

In Region I ( $m>1, p \geqslant 1)$ Darcy's law holds for all interfaces, which can only be of the heating 
type (or stationary for a shorter or larger waiting time). In Region II $(2-m<p<1<m$ ) there exist cooling fronts which obey a different interface equation. The interface dynamics in Region IV $(m>1, m+p<2)$ are controlled by a completely different mechanism. Finally, the line III, $m+p=2$, obeys the modified Darcy law for all kinds of fronts.

Main results. We begin this study with the question of higher regularity of solutions and interfaces. We establish analytic smoothness of the interfaces of compactly supported solutions. We also prove smoothness of the pressure of the solution, the function defined in (1.5). We remark that for the purely diffusive case $b=0$ (i.e. for the porous medium equation), a similar regularity result holds for solutions with suitable initial data, but only for large times if the data are general; cf. [2,7]. Indeed, it can also happen for certain initial configurations that the interface is steady during a finite time interval (the waiting time), at the end of which an angle can be formed in the interface, so that the optimal global regularity is then Lipschitz continuous; cf. [6]. We show here that the waiting-time phenomenon is absent for $b>0$, so that no singularity arises in the interface evolution.

Interesting phenomena occur at the critical points of the interface, which consist of turning points, where the monotonicity of the interface changes, and inflection points, where the concavity changes. We describe a countable set of the special patterns exhibiting turning and inflection-like behaviour of the interfaces. The construction of the patterns (they are not of any self-similar or a group invariant structure) consists of matching three different regions: inner, intermediate and outer regions. We show that in the inner region close to the interface the local inner spatial structure of all the turning/inflection patterns is governed by Kummer's polynomials $K_{l}(\zeta)$ for arbitrary integers $l=2,3, \ldots$, where $\zeta$ is the appropriate spatial rescaled variable. Each pattern is shown to match the outer $(x, t)$-domain via the intermediate region governed by a first-order Hamilton-Jacobi equation.

\section{Preliminaries: interface equation}

A study of the question of regularity of solutions and interfaces of the solutions to this problem is included in the paper [18]. It is known (see the survey [27] and [18]) that the Cauchy problem (1.1) and (1.2) for $p>0$ has a unique continuous weak solution in a finite time interval $0<t<T_{e}$, and that it vanishes identically as $t$ approaches the extinction time $T_{e}$. The Lipschitz continuity of solutions and interfaces is established in the exponent range $m+p \geqslant 2$. If $-m<p \leqslant 0$ then, at least, it admits a unique proper (maximal) solution which is constructed by monotone decreasing regular approximations; see [25] and [24]. If $p \leqslant-m$ then no weak or maximal solution exists and any monotone approximation gives the trivial function $u(x, t) \equiv 0$ for $t>0$ [25] (complete extinction).

The pressure. Taking $a, b>0$, not necessarily 1 , the natural variable to study regularity of solutions with interfaces is the pressure $v(x, t)$ defined as

$$
v=a \frac{m}{m-1} u^{m-1} .
$$

It satisfies the equation

$$
v_{t}=(m-1) v v_{x x}+\left(v_{x}\right)^{2}-a b m \chi_{\{v>0\}} .
$$


It is a $C^{\infty}$-function in the positivity domain where $u>0$ since the equation is uniformly parabolic on any set $\{u \geqslant \delta\}$ with arbitrarily small $\delta>0$. The regularity as $u \rightarrow 0$, when the equation degenerates, is one of the questions under study in this paper.

Estimates. Under the assumption that $u_{0}$ is non-negative, it is known (see [21]) that the pressure $v$ admits an a priori bound for the second space derivative of the form

$$
v_{x x} \geqslant-\frac{1}{(m+1) t},
$$

so-called semi-convexity inequality. It follows that if $v$ is bounded then it also has a bounded derivative in $x$ for $t>0$ :

$$
\left|v_{x}(x, t)\right|^{2} \leqslant \frac{2}{m+1} \frac{\|v(\cdot, t)\|_{\infty}}{t} .
$$

Moreover, (2.3) implies that for every $x_{0} \in \mathbf{R}$ and $t_{0}>0$ there exist lateral derivatives $D_{x}^{-} v\left(x_{0}, t_{0}\right)$ and $D_{x}^{+} v\left(x_{0}, t_{0}\right)$ and

$$
D_{x}^{-} v\left(x_{0}, t_{0}\right) \leqslant D_{x}^{+} v\left(x_{0}, t_{0}\right)
$$

(this is the type of inequality typically found in conservation laws as a form of the entropy conditions). See also [27] and [18].

Finite propagation and interface equation. On the other hand, if the initial data have compact support so does the solution and there appear two outer interfaces defined by

$$
\eta(t)=\inf \{x \in \mathbf{R}: u(x, t)>0\}, \quad \zeta(t)=\sup \{x \in \mathbf{R}: u(x, t)>0\} .
$$

According to the results of [18], for bell-shaped solutions both interfaces, $x=\eta(t)$ and $x=\zeta(t)$ are Lipschitz continuous curves on $\left(\varepsilon, T_{e}-\varepsilon\right), \varepsilon>0$. The present equation represents a simple case (maybe the simplest one) of a non-linear diffusion-absorption equation exhibiting interfaces which do not follow the standard dynamical equation called Darcy's law, which is the well-known interface equation for pure diffusion, i.e. $b=0$. For the right-hand interface Darcy's law reads

$$
D^{+} \zeta(t)=-D_{x}^{-} v(\zeta(t), t)
$$

However, for equation (1.1) with $m>1>p, m+p=2$, the movement of the right-hand interface obeys the equation

$$
D^{+} \zeta(t)=-\left(D_{x}^{-} v-\frac{c}{D_{x}^{-} v}\right)(\zeta(t), t), \quad c=a b m
$$

cf. [18: Theorem 6.2]. Much of the analysis of [18] is based on comparison with the set of travelling wave solutions (TWs), which for $m+p=2$ are given by

$$
v(x, t)=\mu[\lambda t-x]_{+},
$$


where the spatial slope $\mu>0$ and the wave velocity $\lambda \in \mathbf{R}$ are related by the formula

$$
\lambda=F(\mu) \equiv \mu-\frac{a b m}{\mu},
$$

which agrees with (2.7). Clearly $F$ is a one-to-one map from $(0, \infty)$ onto $\mathbf{R}$ so that there exists the explicit inverse function $\mu=\mu(\lambda)>0$ for all $\lambda \in \mathbf{R}$. We call $T_{\lambda}$ the resulting solution with parameter $\lambda$. Observe that we obtain a heating wave $(\lambda>0)$ if $\mu>\mu_{0}=\sqrt{a b m}$, while for $\mu=\mu_{0}$ we get a stationary solution, and for $0<\mu<\mu_{0}$ we have a cooling wave with speed $\lambda<0$. In the limit of small absorption, $b \rightarrow 0$, we see that the branch of cooling wave solutions disappears and the stationary solution becomes flat. In the sequel we put $a=b=1$.

Further regularity. Other results proved in [18] are as follows. The slope $D_{x}^{-} v$ is separated from zero and infinity in the whole interval $\left(0, T_{e}\right)$, uniformly on compact subsets. Moreover, for every $0<t_{0}<T_{e}$ the left derivative $D^{-} \zeta\left(t_{0}\right)$ exists and

$$
D^{-} \zeta\left(t_{0}\right)=\lim _{t \uparrow t_{0}} D^{+} \zeta(t),
$$

while also $D^{+} \zeta\left(t_{0}\right)=\lim _{t \downarrow t_{0}} D^{+} \zeta(t)$. Such a result holds in the more general parameter range $m>1>p, m+p \geqslant 2$. We will show in the next two sections how this regularity can be improved in the critical case $m+p=2$.

\section{3. $C^{1}$-regularity of the interface}

Consider solutions $u(x, t)$ which have exactly two interfaces $\eta(t)<\zeta(t)$ for all $t \in\left(0, T_{e}\right)$ with $u(x, t)>0$ for $\eta(t)<x<\zeta(t)$. For instance, this is true for solutions with bell-shaped initial data having a single maximum. The bell-shapedness is preserved in time since new maximum points cannot occur by the Maximum Principle.

We start the analysis of higher regularity of the interfaces by showing that the solution behaves in an approximately linear way near any interface point for $0<t<T_{e}$. Let us choose a point $\left(x_{0}, t_{0}\right)$ located on the right-hand interface, $x_{0}=\zeta\left(t_{0}\right)$, for $0<t_{0}<T_{e}$. We know that $v_{x}$ is bounded and that (2.3) holds. We prove the following result.

THEOREM 3.1 For every $t_{0} \in\left(0, T_{e}\right)$

$$
D^{+} \zeta\left(t_{0}\right)=D^{-} \zeta\left(t_{0}\right) .
$$

Moreover, near $\left(x_{0}, t_{0}\right)$ we have the estimate

$$
v(x, t)=T_{\lambda_{0}}\left(x-x_{0}, t-t_{0}\right)+o\left(\left|x-x_{0}\right|+\left|t-t_{0}\right|\right),
$$

with $\lambda_{0}=D^{+} \zeta\left(t_{0}\right)$. In particular, the interface slope does not vanish on $\left(0, T_{e}\right)$

$$
D_{x}^{-} v\left(\zeta\left(t_{0}\right), t_{0}\right)=-\mu\left(\lambda_{0}\right) \neq 0 .
$$

We observe that estimate (3.2) of the asymptotic shape, when compared with the TWs, is true for more general equations $[18,19]$. 
Proof. (i) Following the ideas of [5], we perform the scaling

$$
v_{\delta}(x, t)=\frac{1}{\delta} v\left(x_{0}+\delta x, t_{0}+\delta t\right)
$$

with parameter $0<\delta<1$. The idea is to perform an invariant scaling transformation with the parameter $\delta$ around an interface point $\left(x_{0}, t_{0}\right)$ and then let $\delta \rightarrow 0$ (blow-up) in order to study the situation there. It is easy to see that $v_{\delta}$ satisfies the same equation (2.2) in the domain $\mathbf{R} \times$ $\left(-t_{0} / \delta,\left(T_{e}-t_{0}\right) / \delta\right)$. It has the same uniform estimate for the first derivatives since $v_{\delta, x}=v_{x}$ and $v_{\delta, t}=v_{t}$. As for the second derivative, we have

$$
v_{\delta, x x}(x, t) \geqslant-\frac{\delta}{(m+1)\left(\delta t+t_{0}\right)},
$$

where the right-hand side goes to zero as $\delta \rightarrow+0$, an important fact in what follows. Moreover, by [18] the right-hand interface $x=s_{\delta}(t)$ for $v_{\delta}$ passes through the origin with (the right-hand) velocity

$$
D^{+} s_{\delta}(0)=\lambda_{0} .
$$

We also know that there exists the left velocity $D^{-} s_{\delta}(0)=D^{-} \zeta\left(t_{0}\right)=\lambda_{1}$, which can be in principle different from $\lambda_{0}$. Moreover,

$$
\lim _{t \uparrow 0} D^{+} s_{\delta}(t)=D^{-} s_{\delta}(0)=\lambda_{1}, \quad \lim _{t \downarrow 0} D^{+} s_{\delta}(t)=\lambda_{0} .
$$

(ii) Let us now pass to the limit as $\delta \rightarrow+0$. We use the Lipschitz continuity of solutions and interfaces together with known interior regularity to conclude that the family $v_{\delta}$ is compact in a Hölder $(x, t)$-space on compact subsets, so that along a sequence it converges to a certain limit

$$
V(x, t)=\lim _{\delta_{n} \rightarrow 0} v_{\delta_{n}}(x, t) .
$$

It is clear that $V$ is a continuous, weak and non-negative solution of equation (2.2) in the whole plane $\{-\infty<x, t<\infty\}$, and that it has at $t=0$ the linear profile

$$
V(x, 0)=\left[-\mu\left(\lambda_{0}\right) x\right]_{+} .
$$

On the other hand, the second derivative estimate passes to the limit as

$$
V_{x x} \geqslant 0 .
$$

We want to conclude that $V(x, t)$ coincides with $T_{\lambda_{0}}(x, t)$ which ends the proof. The conclusion is obvious for $t>0$ by uniqueness of weak solutions, so we only have to check what happens for $t<0$.

(iii) Let us discuss the limit $V$ for $t<0$. We apply a simple geometric approach based on the semi-convexity estimate. Using (3.6) and the convexity it is easy to see that for $t<0$ the solution $V$ is positive in the region $x-\lambda_{1} t<0$ with interface $x=\lambda_{1} t$ and it has there a slope

$$
D_{x}^{-} V\left(\lambda_{1} t, t\right)=-\mu\left(\lambda_{1}\right),
$$

and in view of the convexity a lower estimate holds:

$$
V(x, t) \geqslant T_{\lambda_{1}}(x, t), \quad t<0 .
$$


This immediately implies, in the limit $t \rightarrow-0$, that $\lambda_{1} \leqslant \lambda_{0}$, since the slope $\mu$ is an increasing function of $\lambda$. We will prove later that the equality holds. For the moment we continue as follows. It is known (and proved by approximation) that the derivative $v_{x}$ of the solutions of equation (2.2) satisfies the Maximum Principle. Since $V_{x}$ attains the value $-\mu\left(\lambda_{0}\right)$ at $t=0$, it follows that $\left\|V_{x}(\cdot, t)\right\|_{\infty}$ was equal or larger than this value for all $t<0$. Now, the function $V(x, t)$ is convex in $x$ for every $t$ so that the maximum value of $-V_{x}$ is taken at $x=-\infty$. It must be precisely $\lambda_{0}$, not larger, otherwise $V$ would resemble a travelling wave with a larger speed and the interface would eventually move faster than $x=\lambda_{0} t$, a contradiction. It follows that

$$
\lim _{x \rightarrow-\infty} V_{x}=-\mu\left(\lambda_{0}\right) .
$$

Suppose now that the equality holds: $\lambda_{1}=\lambda_{0}$. Since $V_{x}$ starts with the value $-\mu\left(\lambda_{1}\right)$ at the interface and it is monotone and ends with value $-\mu\left(\lambda_{0}\right)$, we conclude that $V_{x}$ is constant on the positivity set of $V$ when $\lambda_{0}=\lambda_{1}$, so that $V=T_{\lambda_{0}}$ and the proof is complete when we undo the scaling.

(iv) Next, let us examine the possibility $\lambda_{1}<\lambda_{0}$ and let us arrive at a contradiction. In that case we still have (3.10) and (3.11), and

$$
\mu\left(\lambda_{1}\right) \leqslant-v_{x} \leqslant \mu\left(\lambda_{0}\right)
$$

for $x<-\lambda_{1} t, t<0$. Let us prove next that

$$
V(x, t) \geqslant T_{\lambda_{0}}(x, t)
$$

for $t<0$. We need another estimate, this time on $v_{t t}(x, t)$. It takes the form

$$
\left|v_{t t}\right| \leqslant \frac{C}{\left|x-x_{0}\right|}
$$

valid on small triangular regions of the form

$$
R=\left\{(x, t): x_{0}-\delta<x<x_{0}, 0<2 \lambda_{1}\left(t_{0}-t\right)<\left(x_{0}-x\right)\right\}
$$

with $\delta>0$. This comes from the scaling, arguing by contradiction, on a sequence of points $\left(x_{n}, t_{n}\right) \rightarrow\left(x_{0}, t_{0}\right)$ in $R$, putting $\delta_{n}=x_{0}-x_{n}$, using the uniform convergence of the $v_{\delta}$ and their derivatives, and the equality

$$
v_{\delta, t t}=\delta v_{t t} .
$$

Once this is established, we can derive the estimate

$$
\left|v_{\delta, t t}\right| \leqslant \frac{C}{|x|}
$$

on growing triangles, and in the limit the same inequality is true for $V$ in an infinite triangle backwards in time. Using Taylor's formula at a point $(x, t)$ with $x<2 \lambda_{1} t<0$, we get

$$
V(x, t)=V(x, 0)+t V_{t}(x, 0)+\frac{t^{2}}{2} V_{t t}(x, s) .
$$

The last term is bounded by $C t^{2} / x$. The other two can be computed explicitly at $t=0$ and amount to the travelling wave. We conclude that

$$
V(x, t)-T_{\lambda_{0}}(x, t) \geqslant-\frac{C}{|x|} .
$$


Taking the limit as $x \rightarrow-\infty$ we see that it goes to zero. Since the function is convex, it must be non-negative everywhere.

The final step consists in proving that the equality holds in (3.12). This is an easy consequence of the Strong Maximum Principle since both members are solutions of equation (2.2), which is uniformly parabolic with smooth coefficients in the positivity domain $\{v \geqslant \varepsilon>0\}$. Since both solutions coincide for $t \geqslant 0$ and they are ordered, they must also coincide before. As a summary, this means that the 'initial' data (3.8) prescribe the unique TW solution

$$
V(x, t) \equiv\left[-\mu\left(\lambda_{0}\right)\left(x-\lambda_{0} t\right)\right]_{+}
$$

for any $t \approx 0$.

THEOREM 3.2 The interface functions $\eta(t)$ and $\zeta(t)$ are $C^{1}$ functions for $0<t<T_{e}$. Moreover, the pressure is $C^{1}$ in the positivity set and up to the interfaces, i.e. in

$$
C=\left\{(x, t): 0<t<T_{e}, \eta(t) \leqslant x \leqslant \zeta(t)\right\} .
$$

Finally, $v v_{x x} \rightarrow 0$ as $(x, t) \in\{v>0\}$ approaches a point on one of the interfaces, so that the equation

$$
v_{t}=\left(v_{x}\right)^{2}-m
$$

holds on both interfaces.

Proof. The $C^{1}$ regularity of the interfaces comes from the equality $D^{+} \zeta\left(t_{0}\right)=D^{-} \zeta\left(t_{0}\right)$ which shows differentiability at every time $t_{0}$ for the right-hand interface (the same holds for the left-hand one by symmetry). The continuity of $\zeta^{\prime}(t)$ follows then from the two partial statements

$$
D^{-} \zeta\left(t_{0}\right)=\lim _{t \uparrow t_{0}} D^{+} \zeta(t), \quad D^{+} \zeta\left(t_{0}\right)=\lim _{t \downarrow t_{0}} D^{+} \zeta(t) .
$$

The $C^{1}$ smoothness of the pressure away from the interfaces is standard since the equation is parabolic non-degenerate there. Near the interfaces it follows from the blow-up argument of the previous theorem, as does the fact that $v v_{x x} \rightarrow 0$ as $(x, t)$ approaches the interface, hence the limit equation (3.13). It represents a more classical form of the interface dynamics (2.7). We recall that the pressure has discontinuous gradient across the interfaces, the values involved in formula (3.13) are the inner limits, taken from the positivity set $\{v>0\}$.

\section{Analyticity of the interfaces}

As in the previous section we consider solutions with exactly two interfaces defined for $0<t<T_{e}$, $T_{e}$ being the extinction time.

THEOREM 4.1 The interfaces are real analytic functions for $0<t<T_{e}$.

We adapt the arguments used earlier by Angenent in [2] to establish real analyticity of interfaces in solutions of the one-dimensional porous medium equation (PME). There is a difference in the result: in our present case the interfaces are real analytic functions throughout the interval of existence of the solution $\left(0, T_{e}\right)$, while in the PME case there is a possible exception in the form of a corner point at the waiting time. From the technical point of view, there is a different interface 
equation within the system, cf. (4.5), which forces us to revise the whole argument, although, in the end, it turns out to be valid, moreover zero-slope profiles and the consequent waiting times are eliminated.

Let $v(x, t)$ be the function defined by (1.5). Using notations from [2], we introduce the new unknown

$$
V(\xi, t)=v\left(\frac{\zeta_{+}+\zeta_{-}}{2}+\frac{\zeta_{-}-\zeta_{+}}{2} \cos \xi, t\right), \quad \xi \in \boldsymbol{R}, \quad t \in\left[0, T_{e}-\varepsilon\right],
$$

where we use the more convenient notation $x=\zeta_{+}(t)$ (respectively $x=\zeta_{-}(t)$ ) for the righthand (respectively left-hand) interface. The new function $V(\xi, t)$ can be extended as a $2 \pi$-periodic function of $\xi$ which solves the equation

$$
\begin{aligned}
V_{t}= & \left(\frac{2}{\zeta_{+}-\zeta_{-}}\right)^{2}\left[\frac{(m-1) V}{\sin ^{2} \xi}\left(V_{\xi \xi}-\frac{\cos \xi}{\sin \xi} V_{\xi}\right)+\left(\frac{V_{\xi}}{\sin \xi}\right)^{2}\right] \\
& +\frac{(1+\cos \xi) Z_{-}+(1-\cos \xi) Z_{+}}{\zeta_{+}-\zeta_{-}} \frac{V_{\xi}}{\sin \xi}-m
\end{aligned}
$$

where we have put $Z_{ \pm}(t)=\zeta_{ \pm}^{\prime}(t)$, which are continuous functions according to the results of the previous section. Equation (4.1) differs from that for the PME [2] by the last constant term on the right-hand side. Step by step we will show that this term plays no essential role in both the local and global analyticity analysis.

It follows from the definition of the change of variables $x \mapsto \xi$ that near the line $\xi=0$ (i.e. $\left.x=\zeta_{-}(t)\right)$

$$
V(\xi, t)=v_{x}\left(\zeta_{-}, t\right) \frac{\zeta_{+}-\zeta_{-}}{4} \xi^{2}+o\left(\xi^{2}\right)
$$

Therefore,

$$
\begin{aligned}
& V_{\xi}(0, t)=0 \\
& \frac{V_{\xi}(\xi, t)}{\sin \xi}=v_{x}\left(\zeta_{-}, t\right) \frac{\zeta_{+}-\zeta_{-}}{2}+o(1) \\
& \frac{V(\xi, t)}{\sin ^{2} \xi}=v_{x}\left(\zeta_{-}, t\right) \frac{\zeta_{+}-\zeta_{-}}{4}+o(1) .
\end{aligned}
$$

Similar expansions hold at $\xi=\pi\left(x=\zeta_{+}(t)\right)$.

Hence, since $v_{x}\left(\zeta_{ \pm}(t), t\right)$ is non-zero by (3.3) and therefore $V(\xi, t) / \sin ^{2} \xi>0$ uniformly, equation (4.1) is a non-degenerate parabolic equation with singularities in the coefficients of the first-order terms. Moreover, the free boundaries $x=\zeta_{ \pm}(t)$ become the vertical lines $\xi_{+}=\pi$, $\xi_{-}=0$ in the plane of the new variables $(\xi, t)$.

The equations of the free boundaries can now be derived from equations (2.7), (4.1) and (4.2). We have that

$$
\begin{gathered}
\zeta_{ \pm}^{\prime}(t) \equiv Z_{ \pm}(t)=-\left[v_{x}\left(\zeta_{ \pm}, t\right)-\frac{m}{v_{x}\left(\zeta_{ \pm}, t\right)}\right] \\
V_{\xi \xi}(0, t)=\frac{\zeta_{+}-\zeta_{-}}{2} v_{x}\left(\zeta_{-}, t\right), \quad V_{\xi \xi}(\pi, t)=\frac{\zeta_{+}-\zeta_{-}}{2} v_{x}\left(\zeta_{+}, t\right),
\end{gathered}
$$


whence

$$
\begin{cases}Z_{-}(t)=-Y_{-}+\frac{m}{Y_{-}}, & Z_{+}(t)=-Y_{+}+\frac{m}{Y_{+}}, \\ Y_{-}(t)=\frac{2 V_{\xi \xi}(0, t)}{\zeta_{+}-\zeta_{-}}, & Y_{+}(t)=\frac{2 V_{\xi \xi}(\pi, t)}{\zeta_{+}-\zeta_{-}} .\end{cases}
$$

Problem (4.1), (4.5) is considered as an initial-value problem by defining the triple $w(t)=$ $\left(V, \zeta_{+}, \zeta_{-}\right)$. This function is a solution of a dynamical system of the form

$$
w^{\prime}(t)=F(w(t)) \quad \text { with } \quad F\left(V, \zeta_{+}, \zeta_{-}\right)=\left(V_{t}, Z_{+}, Z_{-}\right) .
$$

In order to consider the problem as an abstract parabolic initial-value problem in the sense of [30], as in [2], we define the following Banach spaces:

$$
\begin{gathered}
E_{0, \theta}=\left\{v \in h^{\theta}(\mathbf{R}): V(\xi) \equiv V(\xi+2 \pi) \equiv V(-\xi), V(0)=V(\pi)=0\right\}, \\
E_{1, \theta}=h^{2+\theta}(\mathbf{R}) \cap E_{0, \theta},
\end{gathered}
$$

where $\theta \in(0,1)$, and $h^{s}(\mathbf{R})$ denotes the little Hölder-space with non-integer exponent $s$. Next, we set

$$
X_{k, \theta}=E_{k, \theta} \oplus \mathbf{R}^{2}, \quad k=0,1,
$$

and consider the open subset $\mathcal{O}_{\theta} \subset X_{1, \theta}$ defined by

$$
\mathcal{O}_{\theta}=\left\{w \in X_{1, \theta} \mid \zeta_{-}<\zeta_{+}, V(\xi)>0 \text { for } 0<\xi<\pi, V_{\xi \xi}(0)>0, V_{\xi \xi}(\pi)>0\right\} .
$$

We thus consider the dynamical system (4.6) with $F: \mathcal{O}_{\theta} \mapsto X_{0, \theta}$. The local analyticity result is obtained by using the general approach to abstract evolution equations [4]. As in [3], it can be shown that $F: \mathcal{O}_{\theta} \mapsto X_{0, \theta}$ is a real analytic map, and that for every $w \in \mathcal{O}_{\theta}$ the Fréchet derivative $\mathrm{d} F(w): X_{1, \theta} \mapsto X_{0, \theta}$, viewed as an unbounded operator in $X_{0, \sigma}(0<\sigma \leqslant \theta)$, generates an analytic semi-group. Since the derivative $\mathrm{d} F$ does not depend on the last constant term $-m$ in equation (4.1), the arguments of [2] apply in this case. Namely, this problem reduces to showing that the operator $A=a(x)(\mathrm{d} / \mathrm{d} x)^{2}+(b(x) / x)(\mathrm{d} / \mathrm{d} x), x>0$, with coefficients $a, a^{-1}, b \in h^{\theta}([0, \infty))$, $a>0$, generates an analytic semi-group in $h^{\theta}([0, \infty))$ if $\operatorname{dom}(A)=\left\{u \in h^{2+\theta}([0, \infty)) \mid u^{\prime}(0)=0\right\}$. This is done in [3] by explicitly constructing the resolvent of the operator $A$.

The next step is to check that $w(t)$ is (locally) real analytic. This can be done by literal repetition of the arguments of [2] based on the approach [30]. If the mapping $F$ smoothly depends on a parameter $\lambda$, so does the solution of the equation $w^{\prime}(t)=F(w)$ [30]. Let us consider the following dependence of $F$ on a real positive parameter $\lambda:(\lambda, w) \mapsto \lambda F(w)$. This is a real analytic map from $\mathbf{R}_{+} \times \mathcal{O}_{\theta}$ to $X_{0, \theta}$. By virtue of the equation for $w(t)$ we also have that $w(\lambda t)$ is a solution of the equation $w^{\prime}(\tau)=\lambda F(w(\tau))$. Thus, $w(\lambda t)$ analytically depends on $\lambda$ in the topology of $C\left([0, T] ; X_{1, \theta}\right)$ with some $T>0$.

The global analyticity analysis on $\left(0, T_{e}\right)$ is performed with a slight modification of the transformations and main arguments in [2]. Let us introduce the local variables near the interface $x=\zeta(t)$ : the new independent space variable will be $y$ defined by $v(x, t)=y^{2} / 2$. This transformation renders the interface $x=\zeta(t)$ the vertical line $y=0$ in the plane of the new 
variables. It is easy to see that the function $p(y, t) \equiv v_{x}$ solves the problem

$$
\begin{aligned}
& p_{t}=\frac{m-1}{2} p^{2}\left(p_{y y}+\frac{m+1}{m-1} \frac{p_{y}}{y}\right)+\frac{m p_{y}}{y} \quad \text { in } \quad(-\eta, \eta) \times\left(0, t^{\prime}\right], \\
& p(t, \eta)=\phi(t), \quad p(y, 0) \text { is given, } \quad p(y, t) \text { is an even function of } y .
\end{aligned}
$$

In this problem $\phi(t)$ is a prescribed $C^{\infty}$ function: $\phi(t)=v_{x}$ along the line $v=\eta$. Notice that $\phi(t)$ is separated away from zero and infinity [18: Theorem 6.2]. In order to rewrite this problem as a problem with homogeneous boundary conditions, we set $p=P+\psi$ where the function $P$ is the new unknown and $\psi$ is an even function satisfying the equation

$$
\psi_{t}=\frac{m-1}{2} \psi^{2} A(\psi) \quad \text { for } \quad t>0, \quad y \in(-\eta, \eta),
$$

where

$$
A=\frac{m-1}{2}\left[\left(\frac{\mathrm{d}}{\mathrm{d} y}\right)^{2}+\frac{m+1}{m-1} \frac{1}{y} \frac{\mathrm{d}}{\mathrm{d} y}\right],
$$

and the same boundary conditions as $p(y, t)$. Such a function $\psi(y, t)$ can be constructed explicitly [2]. For the function $P$ we now have the problem

$$
\begin{gathered}
P_{t}=\frac{m-1}{2}(P+\psi)^{2} A(P)+B(P)+f(P, t) \quad \text { in } \quad(-\eta, \eta) \times\left(0, t^{\prime}\right], \\
P( \pm \eta, t)=0, \quad P(0, t) \text { is given, } \quad P(-y, t)=P(y, t),
\end{gathered}
$$

with the notations

$$
B=\frac{m}{y} \frac{\mathrm{d}}{\mathrm{d} y}, \quad f(P, t)=\frac{m-1}{2}(P+\psi)^{2} A(\psi)+B(\psi)-\psi_{t} .
$$

Finally, we need to prove that any local solution $p$ of problem (4.7) and (4.8) is bounded in $h^{\theta}$. It suffices to show that $v_{x x}$ is bounded because

$$
p_{y}=\left(v_{x}\right)_{y}=v_{x x} x_{y}, \quad \text { and } \quad p=v_{x}=y y_{x}=\frac{y}{x_{y}}, \quad \text { whence } \quad p_{y}=\frac{y v_{x x}}{v_{x}} .
$$

Thus, $p$ is Hölder continuous in $y$ if, say, $v_{x x}$ is bounded. The lower estimate on $v_{x x}$ is given by (2.3).

Lemma 4.1 For every $t_{0} \in\left(0, T_{e}\right)$, there exists an $\varepsilon>0$ such that in the domain $D_{\varepsilon}=$ $\left(l_{\varepsilon}(t), \eta(t)\right) \times\left(t_{0}, t_{0}+\varepsilon\right)$

$$
v_{x x} \leqslant \frac{\delta}{t-t_{0}},
$$

where $x=l_{\varepsilon}(t)$ is the $\varepsilon$-level curve of $v(x, t)$.

Proof. First of all, it can be seen from Theorem 3.2 that for any $t \in\left(0, T_{e}\right)$

$$
\lim _{x \rightarrow \zeta(t)} v v_{x x}=0 .
$$


The function $v_{x x}=W$ satisfies the equation

$$
\mathcal{L} W \equiv W_{t}-(m-1) v W_{x x}-2 m v_{x} W_{x}-(m+1) W^{2}=0 .
$$

Let us consider the function

$$
\omega=\frac{C}{v}+\frac{\delta}{t-t_{0}+\gamma v}
$$

depending on positive constants $C, \gamma, \delta$ to be defined. A straightforward calculation gives

$$
\begin{aligned}
\mathcal{L} \omega & \equiv \frac{m C}{v^{2}}+\frac{C v_{x}^{2}}{v^{2}}+\frac{\delta(m \gamma-1)}{\left(t-t_{0}+\gamma v\right)^{2}}+\frac{(2 m-1) \delta \gamma v_{x}^{2}}{\left(t-t_{0}+\gamma v\right)^{2}} \\
& -\frac{2(m-1) \delta \gamma^{2} v v_{x}^{2}}{\left(t-t_{0}+\gamma v\right)^{3}}-(m+1)\left(\frac{C}{v}+\frac{\delta}{t-t_{0}+\gamma v}\right)^{2} .
\end{aligned}
$$

Making use of the obvious inequality $\gamma v \leqslant t-t_{0}+\gamma v$, we arrive at the inequality

$$
\begin{aligned}
\mathcal{L} \omega & >\frac{\delta(m \gamma-1)}{\left(t-t_{0}+\gamma v\right)^{2}}+\frac{\delta \gamma v_{x}^{2}}{\left(t-t_{0}+\gamma v\right)^{2}}-\frac{2(m+1) C^{2}}{v^{2}}-\frac{2 \delta^{2}(m+1)}{\left(t-t_{0}+\gamma v\right)^{2}}+\frac{m C}{v^{2}} \\
& >\frac{1}{\left(t-t_{0}+\gamma v\right)^{2}}\left[\delta(m \gamma-1)-2 \delta^{2}(m+1)\right]+\frac{C(m-2(m+1) C)}{v^{2}} .
\end{aligned}
$$

To satisfy the condition $\mathcal{L} \omega>0$, it suffices to claim

$$
m \gamma-2 \delta(m+1) \geqslant 1, \quad C \leqslant \frac{m}{2(m+1)} .
$$

Let us now take two level curves of $v(x, t), x=l_{\varepsilon}(t)$ and $x=l_{\sigma}(t), 0<\sigma<\varepsilon$, and define the curvilinear domain $R_{\varepsilon, \sigma}=\left(l_{\sigma}, l_{\varepsilon}\right) \times\left(t_{0}, t_{0}+\varepsilon\right)$ so that

$$
\omega \geqslant W \quad \text { on the parabolic boundary of } R_{\varepsilon, \sigma} .
$$

This choice of $\varepsilon$ (independent of $C$ ) and $\sigma$ is always possible. Indeed, once the parameters $C, \gamma$ and $\delta$ are chosen to satisfy (4.11), it amounts to fulfilling the inequalities

$$
\left.v v_{x x}\right|_{x=l_{\varepsilon}(t)} \leqslant \frac{\delta}{1+\gamma},\left.\quad v v_{x x}\right|_{x=l_{\sigma}(t)} \leqslant C,\left.\quad v v_{x x}\right|_{t=t_{0}} \leqslant \frac{\delta}{\gamma} .
$$

By the Maximum Principle we conclude that

$$
W \leqslant \frac{C}{v}+\frac{\delta}{t-t_{0}+\gamma v} \quad \text { in } \quad R_{\varepsilon, \sigma},
$$

where the constants $C, \gamma, \delta$ are independent of $\sigma$. Hence, this inequality holds in the domain $D_{\varepsilon}$ (the limit of $R_{\varepsilon, \sigma}$ when $\left.\sigma \rightarrow 0\right)$. Next, the constant $C$ is subject to the sole condition $C \leqslant m / 2(m+1)$. Letting $C \rightarrow+0$, we arrive at the desired inequality

$$
W \leqslant \frac{\delta}{t-t_{0}+\gamma v} \leqslant \frac{\delta}{t-t_{0}} .
$$


Repeating the arguments of [3], we now check that problem (4.7) and (4.8) has a local solution $p$ bounded in $h^{\theta}$ which can be continued on $\left(0, t^{\prime}\right]$ for every $t^{\prime}<T_{e}$. It follows then that the local orbit $\left\{V(t), 0 \leqslant t \leqslant T_{e}-\varepsilon\right\}$ is pre-compact in $h^{2+\theta}$ which allows the crucial estimate [2] to extend the analyticity property to any interval $\left(0, t^{\prime}\right)$ with $t^{\prime}<T_{e}$. As we know [21], at $t=T_{e}^{-}$when the interfaces meet, they create a singularity like $\sqrt{T_{e}-t}$. This gives Hölder continuity at $t=T_{e}^{-}$with the exponent $1 / 2$, which is supposed to be the best uniform regularity of the interfaces on closed intervals like $\left[\varepsilon, T_{e}\right]$.

\section{Immediate consequences of analyticity}

\subsection{No waiting time effect}

As an immediate consequence of the analyticity of the interface we arrive at the following conclusion.

LEMMA 5.1 The interfaces do not contain straight-line pieces in the $\{x, t\}$-plane, unless they are globally straight lines.

The possibility of a global straight line can happen, for instance, in the case of travelling waves, but it cannot happen in our diffusion-absorption equation for bounded solutions because of the extinction property. We remark that the property of having a piece of straight line is one of the most peculiar features of the porous medium equation, i.e. the non-absorptive case $b=0$, and it occurs in the form of an interface which does not move from $t=0$ up to a finite waiting time, $t_{*}$, when it begins to move (and then it never stops). This form of metastability is not present for equation (1.1) with $m+p=2$.

\subsection{Finite number of turning and inflection points}

The critical points of $\zeta_{+}(t)$, i.e. the zeros of $\zeta_{+}^{\prime}(t)$, are classified into two groups. When the zero, $t=t_{0}$, is of odd order the sign of $\zeta_{+}^{\prime}(t)$ changes in a neighbourhood of $t_{0}$; we call it a turning point. On the other hand, for an zero of even order, $\zeta$ is monotone in a neighbourhood of $t_{0}$ and it is called an inflection.

LEMMA 5.2 The number of critical points of the function $\zeta_{ \pm}(t)$ is finite on every interval $\left[\tau, T_{e}\right]$, $\tau>0$.

Proof. On the one hand, we check that the zeros of the non-trivial analytic function $\zeta_{ \pm}^{\prime}(t)$ are isolated. This proves the result in intervals of the form $\left[\tau, T_{e}-\tau\right]$. Near the extinction point we use the results of [21].

The result implies the the number of critical points is at most countable on the whole time interval $\left[0, T_{e}\right]$. The number is finite if the initial function is well behaved. For instance, due to the Maximum Principle, this is true if the initial pressure is $C^{1}$ inside the support and its derivarive has a finite number of oscillations.

\section{Countable sets of turning and inflection patterns}

We now study some delicate asymptotic aspects of the interface dynamics. Consider the quasi-linear quadratic equation (2.2) with $a=b=1$ for the pressure (1.5):

$$
v_{t}=\mathbf{A}(v)-m \chi_{\{v>0\}} \equiv(m-1) v v_{x x}+\left(v_{x}\right)^{2}-m \chi\{v>0\} .
$$


For convenience, we will fix the left-hand interface that we call again $x=\eta(t)$. We begin with an exact solution.

\subsection{Example: the first turning pattern}

The first exact solutions describing a turning point of interfaces was constructed by Kersner in [28]. It has a simple truncated inverse parabolic shape

$$
v_{1}(x, t)=\left[C_{0}(t)-C_{1}(t) x^{2}\right]_{+},
$$

where the coefficients $\left\{C_{0}(t), C_{1}(t)\right\}$ solve the following dynamical system:

$$
C_{0}^{\prime}=-2(m-1) C_{1} C_{0}-m, \quad C_{1}^{\prime}=-2(m+1) C_{1}^{2}, \quad t>0 .
$$

This system is integrated explicitly which gives the solution

$$
v_{1}(x, t)=\left[\frac{m+1}{2} t^{1-k}\left(T_{e}^{k}-t^{k}\right)-\frac{x^{2}}{2(m+1) t}\right]_{+}, \quad k=\frac{2 m}{m+1} \in(1,2),
$$

where the finite extinction time $T_{e}>0$ is arbitrary. It is worth mentioning that the exact solution (6.4) is not invariant under any Lie or Lie-Bäcklund group of transformations. See a survey on the results of a general group classification of quasi-linear heat equations in [16] and for this equation in [33]. On the other hand, it is a solution of the equation (6.1) on a two-dimensional subspace $\operatorname{Span}\left\{1, x^{2}\right\}$, which is invariant under the quadratic operator $\mathbf{A}$ and can be constructed by a non-linear method of separation of variables. See [15] and other examples therein.

Consider some other properties of this explicit solution. The most striking property is as follows. Its left-hand interface, which is analytic for $t \in\left(0, T_{e}\right)$

$$
\eta_{2}(t)=-(m+1) \sqrt{t^{2-k}\left(T_{e}^{k}-t^{k}\right)}<0,
$$

is non-monotone and attains the minimum value $X_{1}$ at the moment $T_{1}$

$$
T_{1}=T_{e}\left(\frac{2-k}{2}\right)^{1 / k}, \quad X_{1}=\eta_{2}\left(T_{1}\right)=-(m+1) T_{1} \sqrt{\frac{k}{2-k}} .
$$

Therefore, $t=T_{1}$ is the turning point of the interface. We will show that this turning point corresponds to the first, generic and stable, $K_{2}$-turning pattern of the interface for general solutions, where $K_{2}$ denotes the second Kummer polynomial introduced later on.

At $t=T_{e}^{-}$a new singularity of finite time extinction occurs: the solution vanishes identically. It is curious that the asymptotic behaviour near the extinction time as $t \rightarrow T_{e}^{-}$, exhibited by the above exact solution, is also the stable and generic one. This is proven in [21,22].

\subsection{Asymptotic analysis of patterns in the inner region}

Let us study the situation around a critical point of the left-hand analytic interface of the solution, $x=\eta(t)$. This means that $\eta^{\prime}\left(t_{0}\right)=0$. Using the translational invariance of the PDE, we can put the origin of coordinates at that point and assume that the interface, which is well defined for all $t \approx 0$, 
$\eta(0)=0$, exhibits a special turning or inflection behaviour as $t \rightarrow-0$. In order to classify such a behaviour as $t \rightarrow-0$, we introduce the standard self- similar rescaled variables

$$
v(x, t)=(-t) \theta(\xi, \tau), \quad \xi=x /(-t),
$$

where $\tau=-\ln (-t)$ is the new time variable, so that the finite limit $t \rightarrow-0$ corresponds to the infinite time behaviour, $\tau \rightarrow \infty$, of the rescaled function $\theta(\xi, \tau)$. It satisfies the following quasilinear parabolic equation:

$$
\theta_{\tau}=\mathbf{B}(\theta), \quad \tau \gg 1,
$$

where $\mathbf{B}$ is the quadratic operator

$$
\mathbf{B}(\theta)=\mathbf{A}(\theta)-m \chi_{\{\theta>0\}}+\mathbf{C} \theta, \quad \mathbf{C} \theta=-\theta_{\xi} \xi+\theta .
$$

Obviously, the autonomous equation (6.8) admits the unique non-trivial weak stationary piecewise linear solution with the interface at the origin $x=0$ :

$$
S(\xi)=(\sqrt{m} \xi)_{+} \equiv(\sqrt{m} x)_{+} .
$$

Let us first study the 'non-linear' eigenfunctions which are stationary solutions of the rescaled equation (6.8):

$$
\mathbf{B}(h)=0 .
$$

It is known that in several blow-up problems non-linear eigenfunctions can form finite (see Chapter 4 in [31]) or even infinite countable subsets (see $[12,17]$ and references therein). We show that for the present focusing-like problem, this is not the case.

Proposition 1 Finite interface solutions of equation (6.10) form a one-dimensional set of stationary profiles

$$
h_{A}(\xi)=\left[A \xi+m-A^{2}\right]_{+}, \quad A>0,
$$

with straight monotone interfaces of the travelling-wave type on the $\{x, t\}$-plane. No other solutions exist.

It follows from scaling (6.7), that for any $A \neq \sqrt{m}$ the self-similar solutions describe propagation with a non-zero velocity:

$$
v(x, t) \equiv\left[A x+\left(m-A^{2}\right)(-t)\right]_{+}, \quad \eta^{\prime}(t)=\left(m-A^{2}\right) / A,
$$

and indeed these solutions are the TWs with constant speeds. Thus, non-linear self-similar turning/inflection patterns do not exist.

We now present a new crucial geometric property of such patterns to be studied.

Proposition 2 If $\eta^{\prime}(0)=0$, then for all $\tau \gg 1$ the profile $\theta(\cdot, \tau)$ intersects $S(\xi)$ at least at two points and the corresponding two continuous intersection curves converge to 0 as $\tau \rightarrow \infty$ (i.e. as $t \uparrow 0)$. 
Proof. We give a simple geometric explanation of the above property. Assume for a contradiction, that, for some $\tau_{0}<0$, the profile $\theta\left(\xi, \tau_{0}\right)$ with, say, $\eta\left(\tau_{0}\right)>0$ (the turning case) has a single intersection with $S(\xi) \equiv h_{0}(\xi)$, so that the number of intersections satisfies

$$
I\left(\tau_{0} ; h_{0}\right)=1 .
$$

Then by the Strong Maximum Principle in the positivity domain, this intersection is transversal, i.e. stable upon small $C^{1}$-perturbation of the profiles. By continuity of the family (6.11) upon parameter $A$, for any $A \approx \sqrt{m}, A>\sqrt{m}$, it holds that $I\left(\tau_{0} ; h_{A}\right)=1$. Therefore, at $\tau=\infty$ (i.e. $t=0$ ) the interfaces of both solutions $u(x, t)$ and the corresponding self-similar (TW) solution $V(x, t)=$ $(-t) h_{A}(x /(-t))$ coincide. By a typical intersection comparison conclusion (see Lemma 8.2 in [21]), this means that the unique intersection disappears at $t=0$ so that the solutions are ordered. Namely, $u(x, 0) \geqslant V(x, 0), x \in \mathbf{R}$. By the usual comparison of weak solutions, this implies that for $t \geqslant 0$

$$
\eta(t) \leqslant \frac{m-A^{2}}{A} t,
$$

so that $\eta^{\prime}(0) \leqslant\left(m-A^{2}\right) / A<0$. This contradicts the assumption.

In view of Proposition 1 (which actually means non-existence of non-linear eigenfunctions), we now begin the construction of the 'semi-linear' patterns based on the linearized analysis and a matching procedure. In fact, it is expected that a subset of all the non-linear and semi-linear patterns gives a complete description of all the possible asymptotic structures. We do not discuss this question for the problem under consideration. The question of completeness is quite actual in blow-up problems for quasi-linear reaction-diffusion equations, though it is far from being solved entirely. We note also that the so-called transition phenomena between non-linear and semi-linear spectra for a typical blow-up problem in the delicate case of gradient-dependent diffusivity (where, unlike the current problem, the non-linear spectrum can be countable [17]) is considered in [12].

In the inner domain, to be defined later on according to the appropriate scaling, the turning and inflection patterns can be considered as perturbations about the stationary profile. We perform the linearization transformation

$$
\theta(\xi, \tau)=S(\xi)+Y(\xi, \tau),
$$

where the perturbation $Y(\xi, \tau) \rightarrow 0$ as $\tau \rightarrow \infty$ solves the quasi- linear equation

$$
Y_{\tau}=\mathbf{D} Y+\mathbf{A}(Y), \quad \tau \gg 1,
$$

and $\mathbf{D}$ is the linear operator

$$
\mathbf{D} Y=(m-1) \sqrt{m} \xi Y_{\xi \xi}+(2 \sqrt{m}-\xi) Y_{\xi}+Y .
$$

Setting

$$
\xi=\gamma \zeta, \quad \gamma=(m-1) \sqrt{m},
$$

we obtain the equation

$$
Y_{\tau}=\mathbf{D}_{\mathbf{1}} Y+\gamma^{-2} \mathbf{A}(Y),
$$


where $\mathbf{D}_{\mathbf{1}}$ is the hypergeometric operator

$$
\mathbf{D}_{\mathbf{1}} Y=\zeta Y_{\zeta \zeta}+(N-\zeta) Y_{\zeta}+Y, \quad N=\frac{2}{m-1} .
$$

As $Y(\cdot, \tau) \rightarrow 0$, the quadratic operator $\mathbf{A}$ in (6.16) forms an asymptotically negligible perturbation of the linear degenerate (at $\zeta=0$ ) parabolic flow

$$
Y_{\tau}=\mathbf{D}_{\mathbf{1}} Y .
$$

We observe that this perturbation is non-singular and the perturbed equation inherits a natural interior parabolic regularity. Therefore, the $\Omega$-limit set of equation (6.16) can be studied by standard asymptotic techniques.

The operator $\mathbf{D}_{\mathbf{1}}$ is a standard singular Sturm-Liouville operator of the form

$$
\mathbf{D}_{\mathbf{1}} Y=\frac{1}{\rho}\left(p Y^{\prime}\right)^{\prime}+Y, \quad \eta \in(0, \infty),
$$

with positive coefficients

$$
p(\zeta)=\zeta^{N} \mathrm{e}^{-\zeta}>0, \quad \rho(\zeta)=\zeta^{N-1} \mathrm{e}^{-\zeta}>0, \quad \zeta>0 .
$$

It is not difficult to see that both points $\zeta=0$ and $\zeta=\infty$ are in the limit-point case of singular endpoints [29] and $-\mathbf{D}_{\mathbf{1}}+1$ is semi-bounded below. Therefore, it admits a unique Friedrichs extension [10] which is a self-adjoint operator in the weighted Hilbert space $L_{\rho}^{2}\left(\mathbf{R}_{+}\right)$with the discrete spectrum (see [29] and [8])

$$
\lambda_{l}=l-1, \quad l=0,1,2, \ldots
$$

We observe that after a natural transformation, the value $N=2 /(m-1)$ can be treated as the artificial dimension of an Euclidean space $\mathbf{R}^{N}$ of radial functions and then $L_{\rho}^{2}\left(\mathbf{R}_{+}\right)$is identified with the weighted space $L_{\mu}^{2}\left(\mathbf{R}^{N}\right), \mu=\mathrm{e}^{-\zeta}$.

The eigenfunctions $\left\{K_{l}(\eta)\right\}$ of $\mathbf{D}_{\mathbf{1}}$ are Kummer's functions ( $k$ th order polynomials)

$$
\begin{gathered}
K_{0}(\zeta)=c_{0}, \quad \lambda_{0}=-1 ; \quad K_{1}(\zeta)=c_{1}(-\zeta+N), \quad \lambda_{1}=0 ; \\
K_{2}(\zeta)=c_{2}\left[\zeta^{2}-2(N+1) \zeta+N(N+1)\right], \quad \lambda_{2}=1, \\
K_{3}(\zeta)=c_{3}\left[-\zeta^{3}+3(N+2) \zeta^{2}-3(N+1)(N+2) \zeta+N(N+1)(N+2)\right], \quad \lambda_{3}=2, \text { etc. }
\end{gathered}
$$

They form an orthonormal basis in $L_{\rho}^{2}\left(\mathbf{R}_{+}\right),\left\{c_{l}>0\right\}$ are normalization constants. The unstable subspace and the centre one are one-dimensional: $E^{u}=\operatorname{span}\left\{K_{0}\right\}, E^{c}=\operatorname{span}\left\{K_{1}\right\}$; and the stable subspace $E^{s}$ has co-dimension 2 . We denote

$$
K_{l}(0)=a_{l}>0 .
$$

Each polynomial $K_{l}(\zeta)$ has exactly $l$ zeros for $\zeta>0$ [8].

As usual in the study of singular non-linear phenomena, the unstable manifold for the rescaled equation (6.13) corresponds to the instabilities related to the translational invariance of the original 
parabolic PDE (6.8). The pattern on the centre manifold $W^{c}(0)$ which is supposed to be a tangent to the centre subspace $E^{c}$ at $Y=0$ does not correspond to any turning or inflection pattern since, by Proposition 2, Kummer's function $K_{1}(\eta)$ does not provide us with the minimal number two of zeros for $\zeta>0$ (it has a unique root at $\zeta=N$ ). Obviously, the centre manifold corresponds to the behaviour of strictly monotone interfaces which do not exhibit any kind of turning or inflection singularities.

Thus, the turning/inflection patterns are generated by the evolution orbits on the stable manifold which is spanned by Kummer's polynomials $K_{l}(\zeta)$ with any $l \geqslant 2$. As a first result, we easily check the following.

PROPOSITION 3 Kersner's exact solution (6.4) forms at $t \approx T_{1}$ the minimal shape $K_{2}$-turning behaviour.

The proof is straightforward and relies on the representation (6.4) where we perform translations $x \mapsto-X_{1}+x$ and $t \mapsto T_{1}+t$. In fact, in the rescaled variables, this exact solution belongs to the subspace

$$
W_{3}=\operatorname{span}\left\{1, \zeta, \zeta^{2}\right\}
$$

which is invariant under the quadratic operator $\mathbf{D}_{\mathbf{1}}+\mathbf{A}_{\mathbf{1}}$. Then we arrive at a dynamical system for the coefficients of the expansion of $\theta(\cdot, \tau) \in W_{3}$ (it is equivalent to (6.3)). Solving this dynamical system (or using (6.4)), one can see that the corresponding pattern has the form

$$
Y_{2}(\zeta, \tau)=-C \mathrm{e}^{-\tau} K_{2}(\zeta)+O\left(\mathrm{e}^{-2 \tau}\right),
$$

where $C=C(T)>0$ is a constant. We observe an exponential convergence as $\tau \rightarrow \infty$ to the linear $K_{2}$-pattern. The corresponding interface is given by

$$
\zeta_{2}(\tau)=c \mathrm{e}^{-\tau}+O\left(\mathrm{e}^{-\tau}\right) .
$$

In the original spatial variable $x$ this gives the following interface behaviour:

$$
\eta_{2}(t) \equiv \gamma \zeta_{2}(\tau) \mathrm{e}^{-\tau}=c_{1} \mathrm{e}^{-2 \tau}+O\left(\mathrm{e}^{-3 \tau}\right) \equiv c_{1}(-t)^{2}+O\left((-t)^{3}\right), \quad t \rightarrow-0 .
$$

The analysis for $t>0$ is the same and gives a similar expansion with $(-t)$ replaced by $t$. The constant $c_{1}$ in (6.22) is the same for $t<0$ and for $t>0$. Of course, in view of analyticity of the function (6.5), all the left-hand and right-hand derivatives coincide at the turning moment $t=T_{1}$. The quadratic structure (6.4) of minimal complexity represents the generic stable turning pattern. Other higher mode patterns to be constructed below are expected to be unstable.

REMARK It is curious that the spatial structure of Kummer's functions also plays a key role in the construction of finite non-linear spectra of blow-up patterns for the quasi-linear heat equation $u_{t}=\left(u^{\sigma} u_{x}\right)_{x}+u^{\beta}$ in the parameter range $\sigma>0, \beta>\sigma+1$ of single point blow-up. See [14] and also [31], p. 192.

Turning patterns in the inner region. In order to obtain the $l$ th turning pattern we set

$$
Y=\mathrm{e}^{-\lambda_{l} \tau} W \quad\left(\lambda_{l}=l-1\right),
$$

where $W$ solves the equation

$$
W_{\tau}=\left(\mathbf{D}_{\mathbf{1}}+\lambda_{l}\right) W+\mathrm{e}^{-\lambda_{l} \tau} \gamma^{-2} \mathbf{A}(W)
$$


with an exponentially small perturbation for $\tau \gg 1$. Therefore, passing to the limit $\tau \rightarrow \infty$, one can expect that the $\omega$-limit set of any bounded orbit consists of bounded stationary solutions of the unperturbed equation $\left(\mathbf{D}_{\mathbf{1}}+\lambda_{l}\right) w=0$. Hence, the $l$ th pattern corresponds to the asymptotic behaviour close to the one-dimensional eigenspace of $\mathbf{D}_{\mathbf{1}}$ spanned by $K_{l}: W(\cdot, \tau) \sim$ const $K_{l}(\cdot)$ as $\tau \rightarrow \infty$. This gives the following asymptotic expansion of the solutions to (6.16):

$$
Y_{l}(\zeta, \tau)=-C \mathrm{e}^{-(l-1) \tau} K_{l}(\zeta)+o\left(\mathrm{e}^{-(l-1) \tau}\right), \quad l=2,3, \ldots,
$$

where $C>0$ and $l-1 \equiv \lambda_{l}>0$. In terms of the rescaled variable $\theta$ this reads

$$
\theta_{l}(\zeta, \tau)=\left[\alpha \zeta-C \mathrm{e}^{-(l-1) \tau} K_{l}(\zeta)+o\left(\mathrm{e}^{-(l-1) \tau}\right)\right]_{+}, \quad \alpha=\sqrt{m} \gamma=m(m-1) .
$$

Therefore, the corresponding interface $\zeta_{l}(\tau) \rightarrow 0$ as $\tau \rightarrow \infty$ is obtained from the equation

$$
\alpha \zeta=C \mathrm{e}^{-(l-1) \tau} a_{l}(1+o(1))
$$

and has the form

$$
\zeta_{l}(\tau)=c \mathrm{e}^{-(l-1) \tau}(1+o(1)) .
$$

Finally, in the original spatial $x$-variable the interface of the $l$ th turning pattern takes the form

$$
\eta_{l}(t)=c_{1} \mathrm{e}^{-l \tau}(1+o(1)) \equiv c_{1}(-t)^{l}(1+o(1)), \quad t \rightarrow-0, \quad c_{1}>0 .
$$

Inflection patterns in the inner region. This construction is similar. They correspond to the opposite sign in the linearized solution (6.23) so that the rescaled $l$ th inflection pattern has the form

$$
\theta_{l}(\zeta, \tau)=\alpha \zeta+C \mathrm{e}^{-(l-1) \tau} K_{l}(\zeta)+o\left(\mathrm{e}^{-(l-1) \tau}\right),
$$

where $C>0$. This linearized representation makes sense for $\zeta>0$ only. In order to find the corresponding interface $\zeta_{l}(\tau)<0$ we apply a linear interpolation for $\zeta<0$. Namely, bearing in mind that $K_{l}(\zeta)$ is an $l$ th order polynomial with finite derivative $K_{l}^{\prime}(0)$, we can use the following linear interpolation for $\zeta<0$ :

$$
\theta_{l}(\zeta, \tau) \approx \alpha \zeta+C \mathrm{e}^{-(l-1) \tau} a_{l}(1+o(1))
$$

Thus the interface of the inflection pattern is similar to the corresponding turning one (cf. (6.25))

$$
\eta_{l}(t)=-c_{1} \mathrm{e}^{-l \tau}(1+o(1)) \equiv-c_{1}(-t)^{l}(1+o(1)), \quad t \rightarrow-0, \quad c_{1}>0 .
$$

The existence of such turning/inflection patterns on the stable manifolds tangent to the corresponding one-dimensional stable eigenspaces can be established by a topological method of the so-called conditional invariance (isolating blocks) introduced in [11]; see blow-up applications in [1] and in [9]. In this case we need to establish special bounds on the solutions in the intermediate and outer regions (which are governed by lower-order Hamilton-Jacobi equations), which are quite similar to those on the blow-up profiles for semi-linear and quasi-linear heat equations; [1,9,11].

It is worth mentioning that the analysis above is assumed to be complete in the sense that it gives all possible patterns. Such a conclusion is typical for dynamical systems with such invariant manifolds and is true for some less degenerate blow-up problems; see references in [31], Chapter 4. A general result of this nature is known to play an important role in the zero-set analysis of parabolic equations; see Theorem 4 in [26]. 


\subsection{Intermediate Hamilton-Jacobi region}

It follows from the expansion in the inner region with sufficiently large $\zeta \gg 1$, for which

$$
K_{l}(\zeta)=(-1)^{l} b_{l} \zeta^{l}(1+o(1)), \quad b_{l}>0,
$$

that the linearized turning/inflection patterns take the form

$$
Y_{l}(\xi, \tau)=\mp C_{1} \mathrm{e}^{-(l-1) \tau} \zeta^{l}+o\left(\mathrm{e}^{-(l-1) \tau} \zeta^{l}\right), \quad C_{1}>0 .
$$

We thus define the intermediate region with the new spatial rescaled variable:

$$
y=\zeta \mathrm{e}^{-(l-1) \tau / l} \equiv \gamma^{-1} \xi \mathrm{e}^{-(l-1) \tau / l} \equiv \gamma^{-1} x \mathrm{e}^{\tau / l} .
$$

The linearized solution $Y=Y(y, \tau)$, instead of (6.16), solves the quasi-linear equation

$$
Y_{\tau}=-\frac{1}{l} Y_{y} y+Y+\mathrm{e}^{-(l-1) \tau / l}\left(y Y_{y y}+N Y_{y}\right)+\mathrm{e}^{-2(l-1) \tau / l} \gamma^{-2} \mathbf{A}(Y)
$$

which is a perturbation of the linear Hamilton-Jacobi equation

$$
Y_{\tau}=-\frac{1}{l} Y_{y} y+Y
$$

This non-linear singularly perturbed dynamical system is similar to that studied in [21] and [22]. Passage to the limit $\tau \rightarrow \infty$ is based on the stability theorem [20] for uniformly Lyapunov-stable $\Omega$-limit sets. Then the stationary Hamilton-Jacobi equation

$$
-\frac{1}{l} H_{y} y+H=0
$$

gives the desired family of profiles

$$
H(y)=\mp C_{1} y^{l},
$$

which represent the reduced $\Omega$-limit set of the perturbed equation (6.31).

\subsection{Outer region}

Finally, using a standard compactness argument, see, e.g., Section 8 in [23], we can extend the expansion in the intermediate region to the outer domain corresponding to the original spatial variable $x$ which is supposed to be sufficiently small: $x \approx+0$. Namely, we now calculate the limit-time profile $v(x,-0)(\tau=\infty)$. We need to pass to the limit $\tau \rightarrow \infty$ in the expansion

$$
v(x, t) \equiv(-t) \theta(\xi, \tau)=\mathrm{e}^{-\tau}\left[\sqrt{m} \xi \mp C_{1} y^{l}+\cdots\right] .
$$

Using the spatial scaling variables, one can see that as $\tau \rightarrow \infty$ for any fixed $x \approx+0$

$$
v(x, t)=\mathrm{e}^{-\tau}\left[\sqrt{m} x \mathrm{e}^{\tau} \mp C_{1}\left(\gamma^{-1} x \mathrm{e}^{\tau / l}\right)^{l}+\cdots\right] \rightarrow \sqrt{m} x \mp C_{2} x^{l}+\cdots,
$$

where $C_{2}=C_{1} \gamma^{-l}$. 


\subsection{Extension beyond focusing}

In order to finish our construction of all the turning/inflection patterns, we need to extend the expansions for small $t>0$. Having (6.33), for $t>0$, we arrive at the Cauchy problem with the initial pressure

$$
v(x, 0)=\sqrt{m} x \mp C_{2} x^{l}(1+o(1)), \quad x \geqslant 0 ; \quad l=2,3, \ldots .
$$

We see that as $x \rightarrow+0$ the initial data $v(x, 0)$ is a perturbation of order $O\left(x^{l}\right)$ of the stationary profile $S(x)=(\sqrt{m} x)_{+}$. This determines a slow motion of the interface at $t \approx+0$ (note that $\eta^{\prime}(0)$ must vanish for all the patterns).

The asymptotic analysis as $t \rightarrow+0$ of this initial singularity is easier and can be studied by direct construction of suitable super- and subsolutions. Nevertheless, we present a scheme of analysis which is similar to the above one in order to indicate the main difference between the evolution singularity and the initial one.

The self-similar scaling (cf. (6.7) and (6.15))

$$
v(x, t)=t \theta(\zeta, \tau), \quad \zeta=x / \gamma t, \quad \tau=\ln t \rightarrow-\infty,
$$

with further linearization $\theta(\tau)=S+Y(\tau)$ leads to the quasi-linear equation (6.16) with a different hypergeometric operator (cf. (6.17))

$$
\mathbf{D}_{\mathbf{1}} Y=\zeta Y_{\zeta \zeta}+(N+\zeta) Y_{\zeta}-Y .
$$

Unlike the focusing case of the evolution singularity as $t \rightarrow-0$, this operator has a continuous spectrum. The required expansion with exponential decay as $\tau \rightarrow-\infty$

$$
Y(\zeta, \tau)=\mp C \mathrm{e}^{\mu \tau} \Psi(\zeta)+o\left(\mathrm{e}^{\mu \tau}\right), \quad \mu>0,
$$

follows from the initial condition (6.34)

$$
\Psi(\zeta)=\mp C_{1} \zeta^{l}+\cdots, \quad \zeta \gg 1 .
$$

In the linear approximation, as above, substituting the expansion (6.35) into the equation, we get the equation on the 'eigenvalue' $\mu$ :

$$
\zeta \Psi^{\prime \prime}+(N+\zeta) \Psi^{\prime}-(\mu+1) \Psi=0 .
$$

Among exponentially decaying solutions, it admits solutions with polynomial growth as $\zeta \rightarrow \infty$ of the form $\Psi(\zeta) \sim \zeta^{\mu+1}$. Hence $\mu=l-1>0$. Finally, denoting $\Psi(0)=a_{l}$, the interface behaviour for $t \approx+0$ is described by the approximation (linear interpolation for the inflection patterns)

$$
\alpha \zeta= \pm C \mathrm{e}^{(l-1) \tau} a_{l}(1+o(1)), \quad \tau \rightarrow-\infty,
$$

where

$$
\eta_{l}(t)= \pm c_{1} t^{l}(1+o(1)), \quad t \rightarrow+0, \quad l=2,3, \ldots .
$$

The constants $c_{1}$ in (6.25), (6.28) and (6.36) coincide.

This completes the construction of $K_{l}$ th turning/inflection patterns for any $l=2,3, \ldots$. 


\section{Acknowledgement}

Research supported by TMR networks ERB FMRX CT98- 0201 and ERB FMRX CT97-0157.

\section{REFERENCES}

1. AMADORI, D. Unstable blow-up patterns. Differ. Integr. Equat. 8, (1995) 1977-1996.

2. Angenent, S. Analyticity of the interface of the porous media equation after the waiting time. Proc. Am. Math. Soc. 102, (1988) 329-336.

3. Angenent, S. Local existence and regularity for a class of degenerate parabolic equations. Math. Ann. 280, (1988) 465-482.

4. Angenent, S. Nonlinear analytic semiflows. Proc. R. Soc. Edinburgh 115A, (1990) 91-107.

5. Aronson, D. G., Caffarelli, L. A., \& Kamin, S. How an initially stationary interface begins to move in porous medium flow. SIAM J. Math. Anal. 14, (1983) 639-658.

6. Aronson, D. G., CAfFarelli, L. A., \& VAZQuez, J. L. Interfaces with a corner-point in onedimensional porous medium flow. Comm. Pure Appl. Math. 38, (1985) 375-404.

7. Aronson, D. G. \& VAZQueZ, J. L. Eventual $C^{\infty}$-regularity and concavity of flows in one-dimensional porous media. Arch. Rat. Mech. Anal. 99, (1987) 329-348.

8. Bateman, H. \& ERdÉlyi, E. Higher Transcendental Functions, vol. I. McGraw-Hill, New York (1953).

9. Bebernes, J., Bressan, A., \& Galaktionov, V. A. On symmetric and nonsymmetric blowup for a weakly quasilinear heat equation. Nonl. Differ. Equat. Appl. 3, (1996) 269-286.

10. Birman, M. S. \& Solomjak, M. Z. Spectral Theory of Self-Adjoint Operators in Hilbert Space. D. Reidel, Dordrecht (1987).

11. Bressan, A. Stable blow-up patterns. J. Differ. Equat. 98, (1992) 57-75.

12. Budd, C. \& Galaktionov, V. A. Stability and spectra of blow-up in problems with quasi-linear gradient diffusivity. Proc. Roy. Soc. London A 454, (1998) 2372-2407.

13. Chen, X.-Y., Matano, H., \& Mimura, M. Finite-point extinction and continuity of interfaces in a nonlinear diffusion equation with strong absorption. J. Reine Angew. Math. 459, (1995) 1-36.

14. Elenin, G. G., Kurdyumov, S. P., \& Samarskit, A. A. Non-stationary dissipative structures in a nonlinear heat-conducting medium. USSR Comput. Math. and Math. Phys. 23, (1983) 80-86.

15. GalaKtionov, V. A. Invariant subspaces and new explicit solutions to evolutions equations with quadratic nonlinearities. Proc. Royal Soc. Edin. 125A, (1995) 225-246.

16. Galaktionov, V. A., Dorodnitsyn, V. A., Elenin, G. G., Kurdyumov, S. P., \& Samarskit, A. A. Quasilinear heat conduction equation with source: blow-up, localization, symmetry, exact solutions, asymptotics, structures. J. Soviet Math. 41, (1988) 1222-1292.

17. Galaktionov, V. A., Kurdyumov, S. P., Posashkov, S. A., \& Samarskit, A. A. A non-linear elliptic problem with a complex spectrum of solutions. USSR Comput. Math. and Math. Phys. 26, (1986) $48-54$.

18. Galaktionov, V. A., Shmarev, S. I., \& Vazquez, J. L. Regularity of interfaces in diffusion processes under the influence of strong absorption. Arch. Rat. Mech. Anal. 149, (1999) 183-212.

19. Galaktionov, V. A., Shmarev, S. I., \& VazQuez, J. L. Second-order interface equations for nonlinear diffusion with very strong absorption. Comm. Contemp. Math. 1, (1999) 51-64.

20. Galaktionov, V. A. \& VAzQuez, J. L. Asymptotic behaviour of nonlinear parabolic equations with critical exponents. A dynamical systems approach. J. Funct. Anal. 100, (1991) 435-462.

21. Galaktionov, V. A. \& Vazquez, J. L. Extinction for a quasilinear heat equation with absorption I. Technique of intersection comparison. Comm. Partial. Differ. Equat. 19, (1994) 1075-1106.

22. Galaktionov, V. A. \& VazQuez, J. L. Extinction for a quasilinear heat equation with absorption II. A dynamical systems approach. Comm. Partial. Differ. Equat. 19, (1994) 1107-1137.

23. Galaktionov, V. A. \& VAzQuez, J. L. Blow-up for quasilinear heat equations described by means of nonlinear Hamilton-Jacobi equations. J. Differ. Equat. 127, (1996) 1-40. 
24. Galaktionov, V. A. \& VazQuez, J. L. Necessary and sufficient conditions for complete blow-up and extinction for one-dimensional quasilinear heat equations. Arch. Rat. Mech. Anal. 129, (1995) 225-244.

25. Galaktionov, V. A. \& VazQuez, J. L. Continuation of blow-up solutions of nonlinear heat equations in several space dimensions. Comm. Pure Appl. Math. 50, (1997) 1-68.

26. Henri, D. B. Some infinite-dimensional Morse-Smale systems defined by parabolic partial differential equations. J. Differ. Equat. 59, (1985) 165-205.

27. Kalashnikov, A. S. Some problems of the qualitative theory of non-linear degenerate second-order parabolic equations. Russian Math. Surveys 42, (1987) 169-222.

28. KERSNER, R. On the behaviour of temperature fronts in media with non-linear heat conductivity under absorption. Moscow Univ. Math. Bull. (translation of Vestnik Moskov. Univ. Ser. I Mat. Mekh.) 33, (1978) $35-41$.

29. Levitan, B. M. \& SARgsJan, I. S. Introduction to spectral theory: self-adjoint ordinary differential operators. Transl. Math. Mon. 39, (1975) American Mathematical Society, Providence, RI

30. Da Prato, G. \& Grisvard, P. Equation d'évolutions abstraites de type parabolique. Ann. Mat. Pura Appl. IV(120), (1979) 329-396.

31. Samarskit, A. A., Galaktionov, V. A., Kurdyumov, S. P., \& Mikhailov, A. P. Blow-up in Quasilinear Parabolic Equations. Walter de Gruyter, Berlin (1995).

32. Rosenau, P. \& Kamin, S. Thermal waves in an absorbing and convecting medium. Physica 8D, (1983) 273-283.

33. SVIRSHEVSKII, S. R. The group properties of the model of heat transfer with relaxation of the heat flux. Inst. Prikl. Mat. AN SSSR, Preprint no. 105 (1988) (in Russian). 